OPEN ACCESS

Edited by:

Yingxin Wang,

Tsinghua University, China

Reviewed by:

Tao Peng,

Texas $A$ and $M$ University,

United States

Ying Liu,

Beijing Normal University, China

*Correspondence: Jian Zuo

jian.zuo@cnu.edu.cn

Specialty section:

This article was submitted to

Optics and Photonics,

a section of the journal

Frontiers in Physics

Received: 30 July 2021

Accepted: 04 October 2021

Published: 03 November 2021

Citation:

Li Z, Zuo J, Zhao Y, Han Z, Xu Z,

Zhao $Y$, Yang $C$, Hu $W$ and Zhang $C$ (2021) A Novel Method for the

Enhancement of Composite Materials

Terahertz Image Using Unsharp

Masking and Guided

Filtering Technology.

Front. Phys. 9:750588

doi: 10.3389/fphy.2021.750588

\section{A Novel Method for the Enhancement of Composite Materials' Terahertz Image Using Unsharp Masking and Guided Filtering Technology}

\author{
Zhilong $\mathrm{Li}^{1}$, Jian Zuo ${ }^{1 *}$, Yuanmeng Zhao ${ }^{1}$, Zhongde $\mathrm{Han}^{2}$, Zhihao $\mathrm{Xu}^{2}$, Yunzhang Zhao ${ }^{2}$, \\ Chao Yang ${ }^{3}$, Weidong $\mathrm{Hu}^{2}$ and Cunlin Zhang ${ }^{1}$ \\ ${ }^{1}$ Beijing Key Laboratory for Terahertz Spectroscopy and Imaging, MOE Key Laboratory of Terahertz Optoelectronics, Department \\ of Physics, Capital Normal University, Beijing, China, ${ }^{2}$ Beijing Key Laboratory of Millimeter Wave and Terahertz Technology, \\ Beijing Institute of Technology, Beijing, China, ${ }^{3}$ Kuang-Chi Silver Star Base Technology Room, Shenzhen Kuang-Chi Advanced \\ Technology Co., Ltd, Shenzhen, China
}

When terahertz imaging technology is used for the nondestructive testing of composite materials, the signal is often affected by the experimental environment and internal noise of the system, as well as the absorption and scattering effect of the tested materials. The obtained image has degradation phenomena such as low contrast, poor resolution of small targets and blurred details. In order to improve the image quality, this paper proposes a novel method for the enhancement of composite materials' terahertz image by using unsharp masking and guided filtering technology. The method includes the processing steps of hard threshold shrinkage denoising based on discrete wavelet transform, amplitude imaging, unsharp masking, guided filtering, contrast stretching, and pseudocolor mapping. In this paper, these steps are reasonably combined and optimized to obtain the final resulting image. To verify the effectiveness of the proposed method, a 150-220 GHz high frequency terahertz frequency modulated radar imaging system was used to image three commonly used sandwich structure composites, and the enhancement processing were carried out. The resulting images with significantly enhanced contrast, detail resolution and edge information were obtained, and the prefabricated defects were all detected; Five objective evaluation indexes including standard deviation, mean gradient, information entropy, energy gradient and local contrast were used to compare and analyze the processing results of different image enhancement methods. The subjective and objective evaluation results showed that the proposed method can effectively suppress the noise in terahertz detection signals, enhance the ability of defect detection and positioning, and improve the accuracy of detection. The proposed method in this paper is expected to play a positive role in improving the practicability of terahertz imaging detection technology and expanding its application fields.

Keywords: terahertz imaging, image enhancement, discrete wavelet, hard threshold shrinkage denoising, guided filtering, unsharp masking 


\section{INTRODUCTION}

Terahertz imaging detection technology has the advantages of high penetrability. In the detection of composite materials' defect, it is easier to detect the internal defects of composite materials than other traditional detection technologies, and it can be used for imaging without contacting the tested materials [1]. The photon energy of terahertz wave is in the order of millielectron volts, and it will not cause structural damage to the tested material or produce harmful radiation to biological tissue in terahertz imaging detection [2]. However, in the application of this technology, the terahertz wave will be affected by the experimental environment noise (energy level absorption of water vapor and air molecules around the system), the internal noise of the system (photon radiation noise, thermal noise, granular noise, etc.), and the material properties of the sample to be tested (interlaminar multiple reflections caused by the laminated structure and scattering caused by poor surface roughness of the sample) $[3,4]$, resulting in the degradation of the detection image such as low contrast, low detail resolution and poor sharpness, which affects the application of terahertz imaging detection technology and the accurate judgment of the internal information of the tested material. Thus, the interference properties of terahertz wave in the imaging process are analyzed. Meanwhile, the de-noising technology and image enhancement technology are used to process the detection image, which is expected to obtain the detection image with high contrast and strong detail resolution.

In order to solve the problem of terahertz image degradation, many scholars did a lot of research. Many methods for terahertz image denoising and enhancement were proposed such as mean filtering, Gaussian filtering, nonlocal mean filtering and the edge detection of Laplacian Gaussian operator. Mean filtering has better removal effect on Gaussian noise in the detected image, but the sharpness of the detected image after filtering will be reduced and the image details such as edges will become blurred; Gaussian filtering can effectively filter out speckle noise in the detected image, but it will cause certain damage to the effective information in the detected signal and the edge and texture details of the image; Nonlocal mean filtering can preserve the texture details of the image while filtering out the background noise, but it cannot adaptively select the optimal filtering parameters, resulting in artifacts in the detection image; The edge detection of Laplacian Gaussian operator can effectively enhance the edge and texture details of the detected image, but it will amplify the background noise while enhancing the edge and details of the image, so that the resolution of the detected image cannot be further improved [5-8]. Therefore, it is necessary to develop new terahertz image denoising and enhancement methods. Guided filtering technology was proposed by, after a large number of experiments, which had good performance in image detail enhancement and other aspects [9]. In their research, a guidance image was used to guide the input image, hence the edge of the output image was the same as the input image, and the texture details were similar to the guidance image. It can effectively preserve the edge information while denoising. In addition, Liu et al. and Zhou

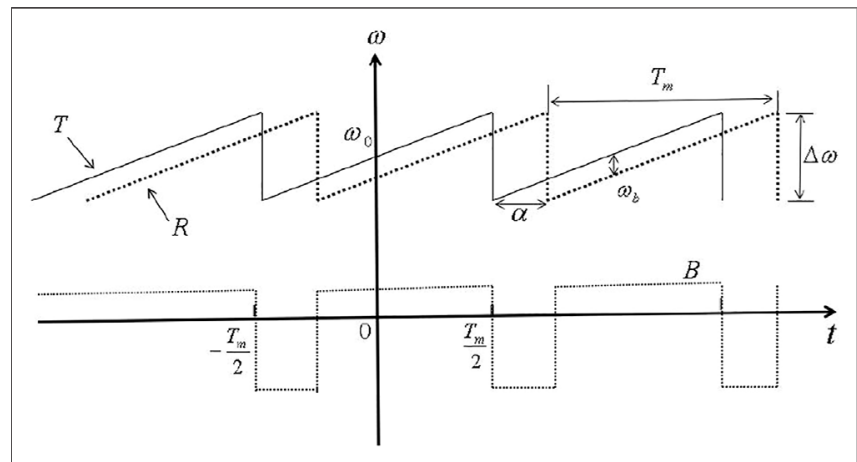

FIGURE 1 | The relationship between angular frequency of the sawtooth wave interference signal and the generated beat frequency signal.

et al. used the guided filtering technology to enhance the details of infrared images, and achieved good results. However, the direct use of guided filtering technology would cause the problem that the background noise of the detection image is amplified [10,11]. Wavelet transform has good time-frequency localization analysis ability and multi-resolution analysis characteristics. In this paper, the hard threshold shrinkage denoising method based on discrete wavelet transform is proposed to filter out the high-frequency noise of the original terahertz detection signal, so as to avoid the problem that the noise in the detection image is amplified after the guided filtering. On the other hand, since the unsharp masking method can preserve the smooth region of the image while highlighting the edge and texture details of the image, this paper proposes to use the sharpened image of the unsharp masking as a guidance image to make the details of the output image clearer. After guided filtering, the obtained detail layer and base layer are gained and superimposed to improve the contrast of terahertz detection image and enhance the detail and edge information of the image. In this paper, a $150-220 \mathrm{GHz}$ high frequency terahertz frequency modulated radar imaging system was used to collect terahertz images of samples, and the denoising and enhancement processing were carried out. In the following, the imaging principle, the principle of wavelet-based unsharp masking and guided filtering method, the experimental samples, experimental setup, the experimental results and objective evaluation are described in detail.

\section{THE PRINCIPLES OF TERAHERTZ FREQUENCY MODULATED RADAR IMAGING SYSTEM}

In this paper, a $150-220 \mathrm{GHz}$ high frequency terahertz frequency modulated radar imaging system had been used for imaging experiments [12]. This system uses a sawtooth wave generator to drive a voltage controlled oscillator to produce a linear frequency modulated continuous wave signal in the microwave band. The instantaneous frequency varies linearly with time in the range of $150-220 \mathrm{GHz}$, and the frequency modulation bandwidth is $70 \mathrm{GHz}$. The relationship between the angular frequency of the interference signal and the generated beat signal in the sawtooth 
wave frequency modulated continuous wave interference is shown in Figure 1.

Among them, solid line $T$ represents the modulated signal directly received by the detector, dotted line $R$ represents the sample signal that carries the sample information after the sample reflection, dotted line $B$ represents the beat frequency signal generated by the interference between the modulated signal and the sample signal, $\Delta \omega$ represents the range of the angular frequency of the modulated signal, $T_{m}$ represents the period of the modulated signal, $\omega_{b}$ represents the frequency difference between the modulated signal and the sample signal, $\alpha$ represents the time delay of the sample signal relative to the modulated signal, and $\omega_{0}$ represents the central angular frequency and is a constant. According to the principle of frequency modulated continuous wave, the longitudinal resolution of the system is expressed as

$$
d=\frac{c}{2 \times \Delta f}
$$

Where $c$ is the speed of light, and $\Delta f$ is the frequency modulation bandwidth of the terahertz source. Therefore, the theoretical longitudinal resolution of the system is $2.1 \mathrm{~mm}$.

When the system is used for terahertz imaging detection of sandwich structure composites with internal defects, the refractive index of defect and non-defect is different, and their reflection coefficient at the interface is different. When the terahertz wave is reflected through the interface, the amplitude of the reflected signal at the defect and non-defect will be different. Therefore, this paper selects the amplitude imaging method that can reflect the specific information of the different interface of the sample to image [13], so as to obtain the internal section information of the sample. The amplitude imaging method is expressed as

$$
S_{d_{0}}(x, y)=S_{\text {Sample }}\left(x, y, d_{0}\right), \quad d_{0} \in\left(d_{1}, d_{2}\right)
$$

Where $S_{d_{0}}(x, y)$ is the terahertz signal set at $d_{0}$ depth within the sample, and its size is $x \times y ; S_{\text {sample }}\left(x, y, d_{0}\right)$ is the terahertz signal set at different depths, and its size is $x \times y \times\left(d_{2}-d_{1}\right) ; d_{1}$, $d_{2}$ are distances between the upper surface and the lower surface of the sample and the detector respectively, and $d_{0}$ are values according to the actual detection requirements.

\section{IMAGE DENOISING ENHANCEMENT BASED ON WAVELET UNSHARP MASKING AND GUIDED FILTERING TECHNOLOGY}

Wavelet transform is a time-frequency analysis method of signal, which has good time-frequency localization analysis ability and multi-resolution analysis function, and can distinguish the abrupt part of signal and noise in different decomposition layers, so it is suitable for analyzing non-stationary signals and extracting the local characteristics of signals [14]. Terahertz detection signal is a typical non-linear and non-stationary signal, so this paper uses the hard threshold shrinkage denoising method based on discrete wavelet transform to pre-denoise the original terahertz detection

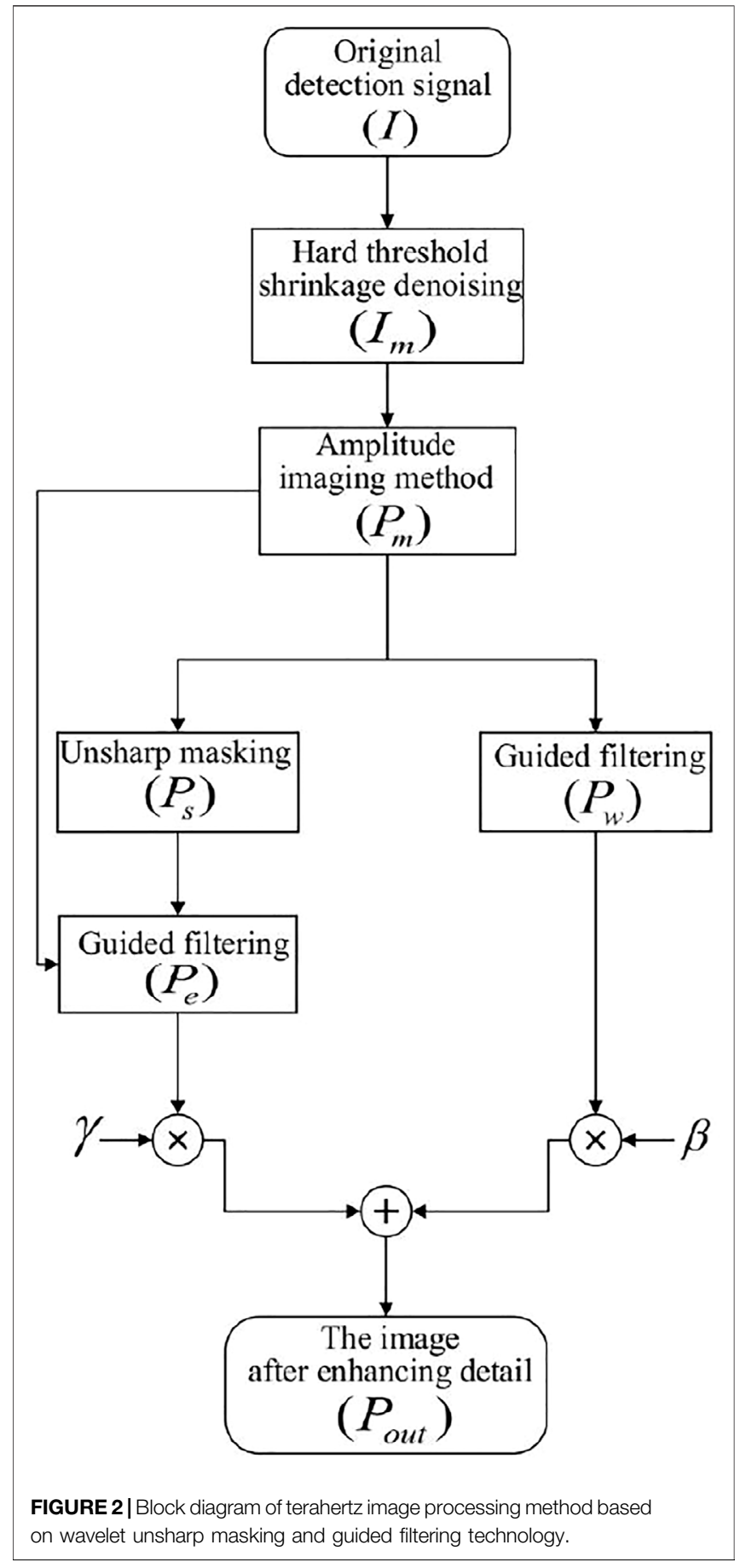

signal, and filter out the high-frequency noise in the detection signal. After the first high frequency denoising, the guided filtering technology which can further denoise and keep the edge information of the image well is introduced to filter the image after wavelet denoising. In order to better enhance the texture detail region of the output image, the sharpened image of the unsharpen masking is used as the guidance image to guide and filter the image after wavelet denoising again; Through the gain and superposition of the detail layer and the base layer 
obtained after filtering, the purpose of improving the contrast of terahertz detection image and enhancing the resolution of image detail is achieved. The block diagram of the method in this paper is shown in Figure 2.

In the first step, the hard threshold shrinkage denoising method based on discrete wavelet transform is used to perform high-frequency denoising preprocessing on the original terahertz detection signal $I$, and the terahertz detection signal $I_{m}$ that filters out most of the highfrequency noise is obtained; In the second step, the amplitude imaging method is used to image the detection signal $I_{m}$ after wavelet denoising, and the preliminary terahertz detection image $P_{m}$ is obtained; In the third step, the guided filtering technique with the image $P_{m}$ after wavelet denoising as the guidance image is used to filter its own image to obtain the detail layer $P_{w}$; At the same time, the unsharp masking based on mean filtering is applied to the denoised images $P_{m}$ to get the sharpened image $P_{s}$; The fourth step is to use the sharpened image $P_{s}$ as the guidance image, to repeat the guided filtering operation, and obtain the base layer $P_{e}$; In the fifth step, the detail layer $P_{w}$ and the base layer $P_{e}$ are gained and superimposed to obtain the terahertz detection image $P_{\text {out }}$ with improved contrast and enhanced detail and edge information. In order to further obtain the detection image with better visualization effect, the pseudo-color mapping of image $P_{\text {out }}$ is carried out by using the color lookup table method, and the contrast and resolution of the imaging image are enhanced by stretching the contrast and adjusting the scale data range and color scale of the mapping, so as to obtain the final detection resulting image.

\section{Hard Threshold Shrinkage Denoising Enhancement Based on Discrete Wavelet Transform}

For a square integrable function $f(t) \in L^{2}(R), \psi(t)$ is the continuous wavelet generator function, $\psi_{a, b}(t)$ is the continuous wavelet basis function, and the continuous wavelet transform of $f(t)$ is defined as

$$
H_{f}(a, b)=\left\langle f(t), \psi_{a, b}(t)\right\rangle=a^{-1 / 2} \int_{-\infty}^{+\infty} f(t) \overline{\left(\frac{t-b}{a}\right)} d t, \quad a>0
$$

Where $H_{f}(a, b)$ is the result of $f(t)$ continuous wavelet transform, $a$ is the scale factor, $b$ is the time shift factor, and $\psi \overline{(} \cdot)$ is the conjugate of the wavelet generating function. Taking $a=a_{0}^{j}, b=a_{0}^{j} k$ ( $j$ is the discretization index, $k$ is the discretization coefficient, $j, k \in Z$ ), the two are discretized, and the discrete wavelet transform can be defined as

$$
H_{f}(j, k)=\left\langle f(t), \quad \psi_{j, k}(t)\right\rangle=a_{0}{ }^{-\frac{j}{2}} \int_{-\infty}^{+\infty} f(t) \overline{\left(a_{0}^{j}-k\right)} d t
$$

Where $H_{f}(j, k)$ is the result of discrete wavelet transform of $f(t)$ $[15,16]$.

In the detection of the sample under test, the detected signal usually contains certain noise, which can be considered as
Gaussian white noise. The signal containing noise can be simply expressed as follows

$$
y_{j}=f_{j}+\delta \cdot z_{j}, \quad j=1, \ldots, n
$$

Where $y_{j}$ is the signal containing noise, $f_{j}$ is the ideal noise-free signal, $z_{j}$ is the doped noise, $\delta$ is the noise level, and $n$ is the sampling length. The essence of signal denoising is to use the different characteristics of signal and noise to extract noise $\delta \cdot z_{j}$ from noise containing signal $y_{j}$ and restore the ideal signal $f_{j}$. For the ideal effective signal, it is continuous in the time domain. Therefore, after the discrete wavelet transform, the energy of the effective signal is mainly concentrated in the low frequency subband, and the modulus of the generated wavelet coefficient would be relatively large. For Gaussian white noise, there is no continuity in the time domain, showing a strong randomness. Meanwhile, after conducting discrete wavelet transform, it also has a strong randomness, thus the energy of noise in wavelet domain is mainly concentrated in the high frequency subband, often still think of the Gaussian white noise, produced by the modulus of wavelet coefficient will be relatively small. Based on the above characteristics, the wavelet coefficients corresponding to noise are still satisfy the Gaussian white noise distribution. According to the definition of standard deviation and variance of random signals, the standard deviation of signals reflects the dispersion degree from each point in the discrete signal to the mean value of signals. Therefore, this paper proposes to take the standard deviation of wavelet coefficients of noise containing signals under the decomposition of each scale of discrete wavelet transform as the threshold to reflect the distribution range of noise wavelet coefficients to a certain extent.

Define the scale function $\varphi(t) \in L^{2}(R)$ by an integer multiple translation $k$ to get $\varphi_{0, k}(t)$, then

$$
\varphi_{0, k}(t)=\varphi(t-k), k \in Z
$$

If space $V_{0}=\overline{\operatorname{Span}\left\{\varphi_{0, k}(t)\right\}}$ is defined by a signal linearly expressed by signal $\varphi_{0, k}(t)$, then $V_{0}$ is called a closed signal space of $\varphi_{0, K}(t)$.By translating and stretching the scale function, a new scale function $\varphi_{j, k}(t)$ is obtained, which is expressed as

$$
\varphi_{j, k}(t)=2^{j / 2} \varphi\left(2^{j} t-k\right), \quad j \in Z, \quad k \in Z
$$

Similarly, the newly closed signal space spanned by $\varphi_{j, k}(t)$ is

$$
\begin{aligned}
\varphi_{j, k}(t) & =V_{j}=\overline{\operatorname{Span}_{k}\left\{\varphi_{j, k}(t)\right\}} \\
& =\frac{\operatorname{Span}_{k}\left\{2^{j / 2} \varphi\left(2^{j} t-K\right\}, j \in Z, k \in Z\right.}{}
\end{aligned}
$$

After further derivation of the scale function $\varphi_{j, k}(t)$, the multiresolution analysis equation corresponding to the scale function coefficient is obtained, and the expression is

$$
\varphi(t)=\sum_{k} h_{0}[k] \cdot \sqrt{2} \varphi(2 t-k)
$$

The equation shows that different scale functions correspond to different $h_{0}[k][17,18]$. When the noise containing signal $y_{i} \in V_{j+1}$, the noise containing signal can be expressed as 


$$
y_{i}=\sum_{k} c_{j}[k] \cdot \varphi_{j, k}(t)+\sum_{k} d_{j}[k] \cdot \psi_{j, k}(t)
$$

Among them, there is

$$
c_{j}[k]=\left\langle y_{i}, \varphi_{j, k}(t)\right\rangle, \quad d_{j}[k]=\left\langle y_{i}, \psi_{j, k}(t)\right\rangle
$$

According to the multi-resolution analysis equation $\varphi_{t}=\sum_{k} h_{0}[n] \cdot \sqrt{2} \varphi(2 t-n)$ of the scale function, we can obtain

$$
\varphi_{j, k}(t)=\sum_{n} h_{0}[n-2 k] \cdot \varphi_{j+1, n}(t)
$$

Then there is

$$
\begin{aligned}
c_{j}[k] & =\left\langle y_{i}, \varphi_{j, k}(t)\right\rangle=\sum_{n} h_{0}[n-2 k] \cdot\left\langle y_{i}, \varphi_{j+1, n}(t)\right\rangle \\
& =\sum_{n} h_{0}[n-2 k] \cdot c_{j+1}[n]
\end{aligned}
$$

Similarly, according to $\psi(t)=\sum h_{1}[n] \cdot \sqrt{2} \varphi(2 t-n)$, the wavelet coefficient $d_{j}[k]$ at each scale and each decomposition layer can be obtained as

$$
d_{j}[k]=\sum_{n} h_{1}[n-2 k] \cdot c_{j+1}[n]
$$

Set the standard deviation of the wavelet coefficient $d_{j}[k]$ as threshold $U$, and the expression is

$$
U=\sqrt{\frac{1}{M-1} \sum_{q=1}^{M}\left\{\left(\sum_{n} h_{1}[n-2 k] \cdot c_{j+1}[n]\right)_{q}-\frac{\sum_{q=1}^{M}\left(\sum_{n} h_{1}[n-2 k] \cdot c_{j+1}[n]\right)_{q}}{M}\right\}^{2}}
$$

where $M$ is the number of wavelet coefficients of the noise containing signal under the decomposition of each scale of the discrete wavelet transform.

According to the characteristics of Gaussian distribution, setting the wavelet coefficient within the threshold range to zero can suppress the interference of noise to the maximum extent, namely, the effect of hard threshold function. Therefore, this paper selects the hard threshold function to carry out the hard threshold shrinkage denoising process for the wavelet coefficient of the noise containing signal. When the absolute value of the wavelet coefficient of the noise containing signal is less than the set threshold, it is made to be zero; otherwise, it keeps unchanged. After shrinkage denoising, the wavelet coefficients are reconstructed and restored to the terahertz detection signal, and the amplitude imaging method is used for imaging processing to obtain the preliminary terahertz detection image.

\section{Guided Filtering}

Guided filtering [9-11] is a filtering method with good performance in image denoising, defogging and detail enhancement. Its core idea is to guide the input image through a guidance image based on the local linear model, so that the overall contour feature of the output image is similar to that of the input image, and the texture detail region is similar to that of the guidance image. The output image $q$ can be expressed as follows

$$
q=f_{\text {guider }}(p, I, r, \varepsilon)
$$

Where $p$ is the input image, i.e., the image after wavelet denoising, $I$ is the guidance image, $r$ is the size of the filtering window, $\varepsilon$ is the regularization parameter, $r$ and $\varepsilon$ can be determined by empirical value. In this paper, $r$ is 3 and $\varepsilon$ is 0.64 . The assumption between output image $q$ and guidance image $I$ is as follows : there is a linear relationship in local window $w_{\mathrm{v}}$ centered on pixel $v$, and the output expression of a pixel can be expressed as

$$
q_{i}=g_{v} I_{i}+o_{v}, \quad \forall i \in w_{v}
$$

Where $i$ corresponds to the pixel index in the local window, $g_{v}$ and $o_{v}$ are linear coefficients in the window. By calculating the derivative gradient on both sides of the above equation, $\nabla q=g \nabla I$ can be obtained. It can be seen that when the input guidance image has gradient in a certain region, the output image will also maintain the corresponding gradient. Therefore, the guided filter has good edge preservation performance while smoothing the background. In order to obtain the optimal solution of $g_{v}$ and $o_{v}$, it is necessary to retain the effective information of the input image $p$ as much as possible in the output image $q$. Even if the difference between the two is the smallest, the method is usually to introduce the minimization cost function and minimize it to solve the optimization problem. $E\left(g_{v}, o_{v}\right)$ is the minimization cost function, and the expression is as follows

$$
E\left(g_{v}, o_{v}\right)=\sum_{i \in w_{v}}\left[\left(g_{v} I_{i}+o_{v}-p_{i}\right)^{2}+\varepsilon g_{v}^{2}\right]
$$

Where $\varepsilon g_{v}^{2}$ is the penalty term, $\varepsilon$ is used to prevent $g_{v}$ too large regularization parameters. The Eq. $\mathbf{1 8}$ is solved by linear regression model to minimize it, and the optimal solution of $g_{v}$ and $o_{v}$ can be obtained as

$$
\begin{gathered}
g_{v}=\frac{\frac{1}{|w|} \sum_{i \in w_{v}} I_{i} p_{i-} \mu_{v} \overline{p_{v}}}{\sigma_{v}^{2}+\varepsilon} \\
o_{v}=\overline{p_{v}}-g_{v} \mu_{v}
\end{gathered}
$$

Where $\mu_{v}$ and $\sigma_{v}^{2}$ are the mean value and variance of pixels in local window $w_{v}$ of the guidance image, $|w|$ is the number of pixels contained in $w_{v}$, and $\overline{p_{v}}=\frac{1}{|w|} \sum_{i \in w_{v}} p_{i}$ is the mean value of image $p$ to be filtered in the window. When using Eq. 19 to calculate the linear coefficient, the $g_{v}$ and $o_{v}$ calculated in different window $w_{v}$ are obviously different, which can be solved by averaging the values obtained from $g_{v}$ and $o_{v}$, so the output image can be expressed as

$$
q_{i}=\frac{1}{|w|} \sum_{i \in w_{k}}\left(g_{v} I_{i}+o_{v}\right)=\overline{g_{i}} I_{i}+\overline{o_{i}}
$$

Where $\overline{g_{i}}=\frac{1}{|w|} \sum_{\nu \in w_{v}} g_{v}$ and $\overline{o_{i}}=\frac{1}{|w|} \sum_{v \in w_{v}} o_{v}$ are the average of linear coefficients of all local windows under the same pixel index. 
Guided Filtering Based on Unsharp Masking Unsharp masking $[19,20]$ is a common sharpening enhancement technique. Its basic principle is that the high frequency part of the image is obtained by subtracting the original image from the blurred image that is obtained by low-pass filtering, and then the high frequency part of the image is gained and superimposed with the original image to obtain the resulting image with enhanced details and edges. The unsharp masking process can be expressed as follows

$$
l(i, j)=f(i, j)+\zeta \times\{f(i, j)-L P[f(i, j)]\}
$$

where $f(i, j)$ is the original input image, $L P$ is the low-pass filter, $\zeta$ is the gain coefficient of high-frequency details, and $l(i, j)$ is the enhanced output image.

In this paper, the mean filter is selected as the low-pass filter smoothing filter, and the mean filter template with a size of $3 \times 3$ is generated to conduct convolution filtering on the image after wavelet denoising, so as to obtain its blurred image. According to the principle of unsharp masking, the difference operation between the original image and the blurred image is used to obtain the mask image, that is, the image reflecting the detail information of the image. After $\zeta$ factor multiplication of the mask image, it is superimposed with the original image to obtain the sharpened image with enhanced detail and edge information in the image after wavelet denoising, so as to achieve the effect of image sharpening. Using this linear unsharp masking method to process the terahertz detection image can get better sharpening effect. While the sharpness of the details is improved, the smooth region in the original image is not affected and preserved. Finally, the sharpened image of unsharp masking is used as the guidance image to conduct the guided filtering on the image after wavelet denoising, so that the texture details in the image after wavelet denoising are similar to those in the guidance image.

Image $P_{m}$ after wavelet denoising is processed by guided filtering technology with its own image $P_{m}$ and sharpened image $P_{s}$ as the guidance image, the detail layer $P_{w}$ reflecting the image detail information and the base layer $P_{e}$ reflecting the image contour are obtained. The obtained detail layer $P_{w}$ and the base layer $P_{e}$ are superposed with the gain of $\beta$ and $\gamma$ coefficients respectively, and the terahertz detection resulting image $P_{\text {out }}$ with improved contrast, enhanced detail and edge information is obtained. The process can be expressed as follows

$$
P_{\text {out }}=\beta \times P_{w}+\gamma \times P_{e}
$$

Where $P_{\text {out }}$ is the output terahertz detection resulting image, $\beta$ and $\gamma$ are the gain coefficients, $P_{w}$ is the detail layer, and $P_{e}$ is the base layer.

\section{IMAGING SAMPLES, EXPERIMENTAL METHODS AND IMAGE PROCESSING}

\section{Sample Introduction and Experimental Setup}

Polymethacrylimide foam composites are heat-resistant composites with the highest strength and stiffness to weight ratio. As the core material of sandwich structure, polymethacrylimide foam composites are widely used in aerospace, shipbuilding, military and other fields [21]. As one of the three major polymer materials, rubber has high elasticity than metal, high mechanical strength and good bending resistance. Because of its unique properties, rubber is widely used in medical and health, power communication, civil engineering and other fields [22]. Glass fiber reinforced composites are widely used in the bearing and electromagnetic wave transmission structures of unmanned aerial vehicles due to their excellent strength-weight ratio, aerodynamic performance and microwave permeability [23]. With the increasingly wide application of the above composites, the demand for its quality control, nondestructive testing and evaluation is also increasing. In the process of preparation and application, the above composites will be affected by external forces and temperature variations, resulting in internal defects such as cracks, voids and adhesive debonding, affecting the normal use of composite materials, and when the damage reaches a certain level, it will even cause a catastrophe [24], so the accurate detection of composite materials has important practical significance.

\section{Polymethacrylimide Foam Sandwich Structure Composite Material}

In this paper, aramid fiber paper honeycomb and polymethacrylimide foam composites were used to combine into sample 1 (the actual photos, defect location and size, structure diagram were shown in Figure 3). The sample was composed of thin aramid fiber paper honeycomb with thickness of $4.0 \mathrm{~mm}$, two layers of polymethacrylimide foam with thickness of $4.3 \mathrm{~mm}$ and aramid fiber paper honeycomb with thickness of $40.0 \mathrm{~mm}$, which were bonded to each other by epoxy resin adhesive, and two layers of Teflon films with diameters of 10.0 and $6.0 \mathrm{~mm}$ and thicknesses of $0.1 \mathrm{~mm}$ were used to prefabricate the adhesive debonding defects of different sizes in the sample. The locations of the two adhesive debonding defects were shown in the green mark in Figure 3.

\section{Rubber and Glass Fiber Reinforced Composite Sandwich Structure}

Sample two rubber and glass fiber reinforced composite sandwich structure (the actual photos, defect location and size, structure diagram were shown in Figure 4). The sample consisted of a rubber of $10.0 \mathrm{~cm} \times 10.0 \mathrm{~cm}$ in size and $5.0 \mathrm{~mm}$ in thickness and a glass fiber reinforced composite of the same size and $13.0 \mathrm{~mm}$ in thickness. The two were bonded to each other by epoxy resin adhesive, and three delamination defects with a length of $4.2 \mathrm{~cm}$ and a depth of $2.0 \mathrm{~mm}$ were prefabricated in the glass fiber reinforced composite. The locations of the defects were shown in the yellow block in Figure 4.

\section{Polymethacrylimide Foam and Polyvinyl Chloride Sheet Sandwich Composite Material}

Sample 3 polymethacrylimide foam and polyvinyl chloride sheet sandwich composite material (the actual photos, defect location and size, structure diagram were shown in Figure 5). The sample was composed of two layers of $4.3 \mathrm{~mm}$ in thickness of 
A

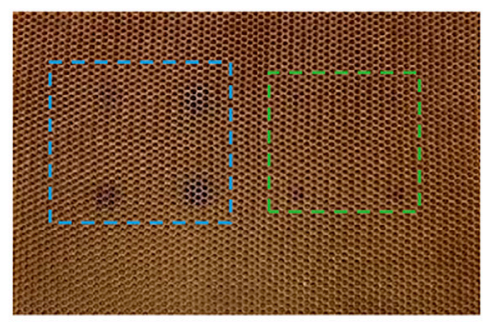

C

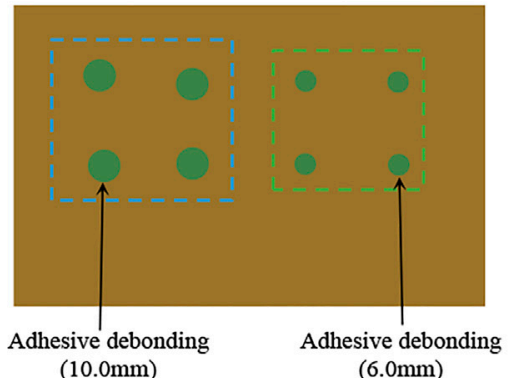

B

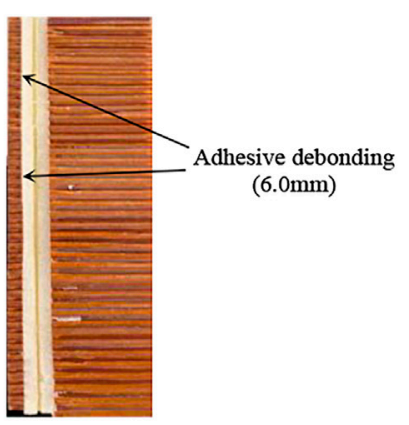

D

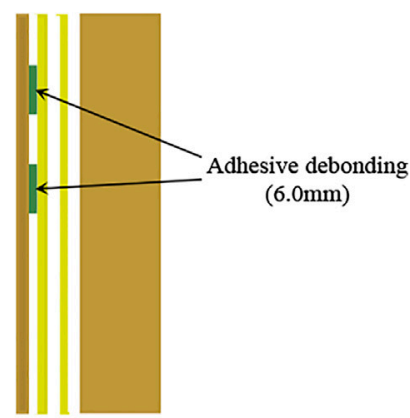

E

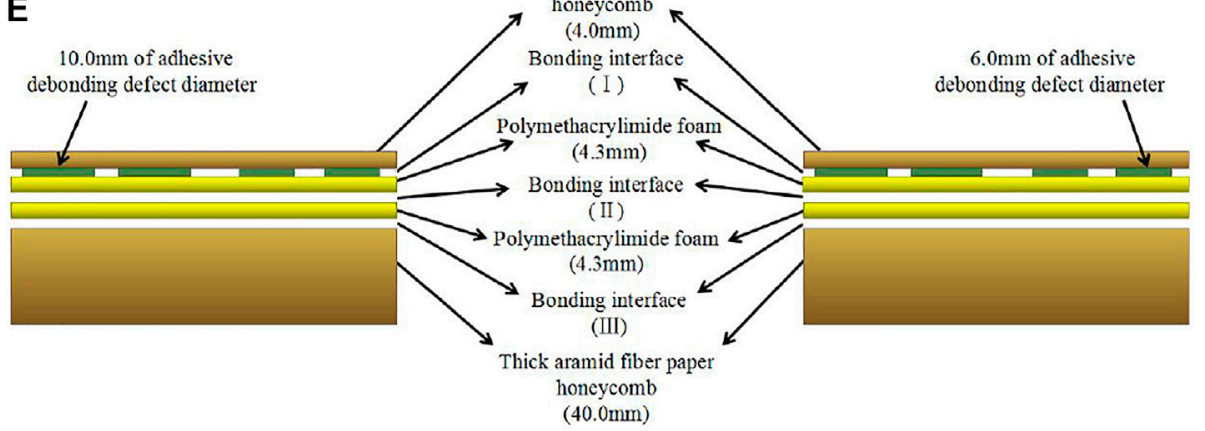

FIGURE 3 | The actual photos, defect location and size, structure diagram of aramid fiber paper honeycomb and polymethacrylimide foam sandwich composite material. (A) the actual photo (top view), (B) the actual photo (side view), (C) the diagram of defect location and size (top view), (D) the diagram of defect location and size (side view), (E) the structure diagram.

polymethacrylimide foam and a layer of $2.0 \mathrm{~mm}$ in thickness of polyvinyl chloride sheet, which were also bonded with epoxy resin adhesive, and Teflon films of 10.0 and $3.0 \mathrm{~mm}$ in diameter and $0.1 \mathrm{~mm}$ in thickness were respectively used to prefabricate the adhesive debonding defects of different sizes in the sample. The locations of the two adhesive debonding defects were shown in the green mark in Figure 5.

\section{Experimental Setup}

In this paper, when the $150-220 \mathrm{GHz}$ high frequency terahertz frequency modulated radar imaging system was used for imaging detection of the above composite materials, in order to reduce the system noise and make the detection images clearer, all samples were placed near the focal plane of the detection system and detected by one-way point-by- point scanning. In order to further improve the detection accuracy of the detection system, the minimum detection accuracy of the stepper motor for the adhesive debonding defect in sample 1 with a diameter of $10.0 \mathrm{~mm}$ was set as $2.0 \mathrm{~mm} \times 2.0 \mathrm{~mm}$, and the minimum detection accuracy of the stepper motor for the adhesive debonding defect with a diameter of $6.0 \mathrm{~mm}$ was set as $1.0 \mathrm{~mm} \times 1.0 \mathrm{~mm}$; The minimum detection accuracy of the stepper motor was set as $1.5 \mathrm{~mm} \times 1.5 \mathrm{~mm}$ for the delamination defects in sample two; the minimum detection accuracy of the stepper motor for the adhesive debonding defect in sample 3 with a diameter of $10.0 \mathrm{~mm}$ was also set as $1.5 \mathrm{~mm} \times 1.5 \mathrm{~mm}$, and the minimum detection accuracy of the stepper motor for the adhesive debonding defect with a diameter of $3.0 \mathrm{~mm}$ was set as $1.0 \mathrm{~mm} \times 1.0 \mathrm{~mm}$. 
A

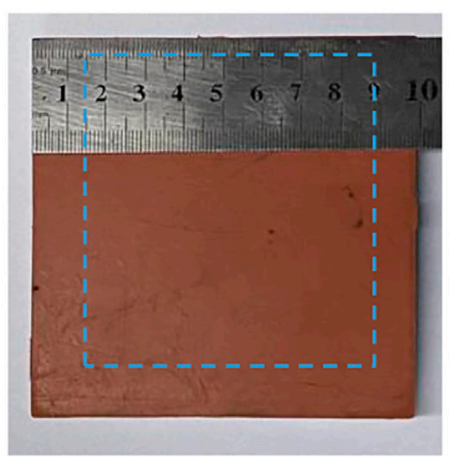

C

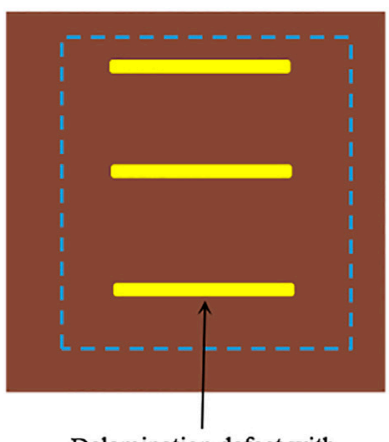

Delamination defect with length of $4.2 \mathrm{~cm}$
B

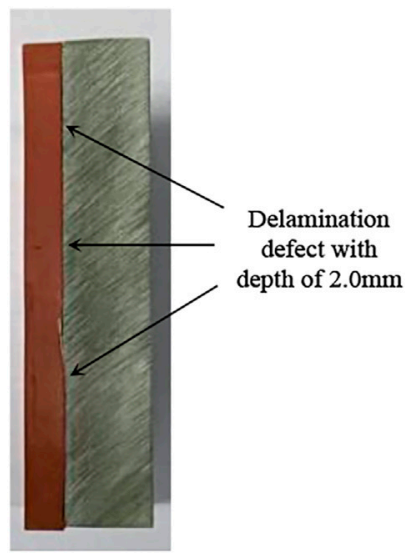

D

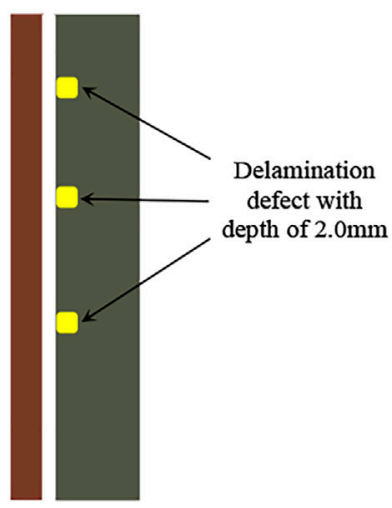

E

Delamination defect with depth of $2.0 \mathrm{~mm}$

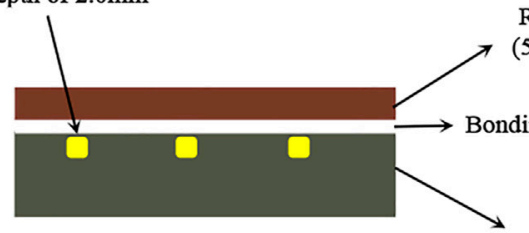

Rubber

Delamination defect with length of $4.2 \mathrm{~cm}$

Glass fiber reinforced composite

(13.0mm)

FIGURE 4 | The actual photos, defect location and size, structure diagram of rubber and glass fiber reinforced composite sandwich structure. (A) the actual photo (top view), (B) the actual photo (side view), (C) the diagram of defect location and size (top view), (D) the diagram of defect location and size (side view), (E) the structure diagram.

\section{Image Processing Results and Analysis}

After the imaging detection of the above composite materials, the amplitude imaging method was used to image the obtained terahertz detection signal, and Gaussian filtering and the method proposed in this paper were used to denoise and enhance the terahertz detection image. When the hard threshold shrinkage denoising method based on discrete wavelet transform was used to denoise the original detection signal, for the adhesive debonding defect with a diameter of $10.0 \mathrm{~mm}$ in sample 1, "sym4" was selected as the wavelet denoising combination with 3 layers of decomposition layer, and "sym5" was selected as the wavelet denoising combination with four layers of decomposition layer for the adhesive debonding defect with a diameter of $6.0 \mathrm{~mm}$; For the delamination defects in sample 2, "sym5" was selected as the wavelet denoising combination with two layers of decomposition layer; For the adhesive debonding defect with a diameter of $10.0 \mathrm{~mm}$ and the adhesive debonding defect with a diameter of $3.0 \mathrm{~mm}$ in sample 3, the combination of wavelet denoising with "sym5" as the wavelet basis and the decomposition layer of 3 layers was selected for hard threshold shrinkage denoising. 


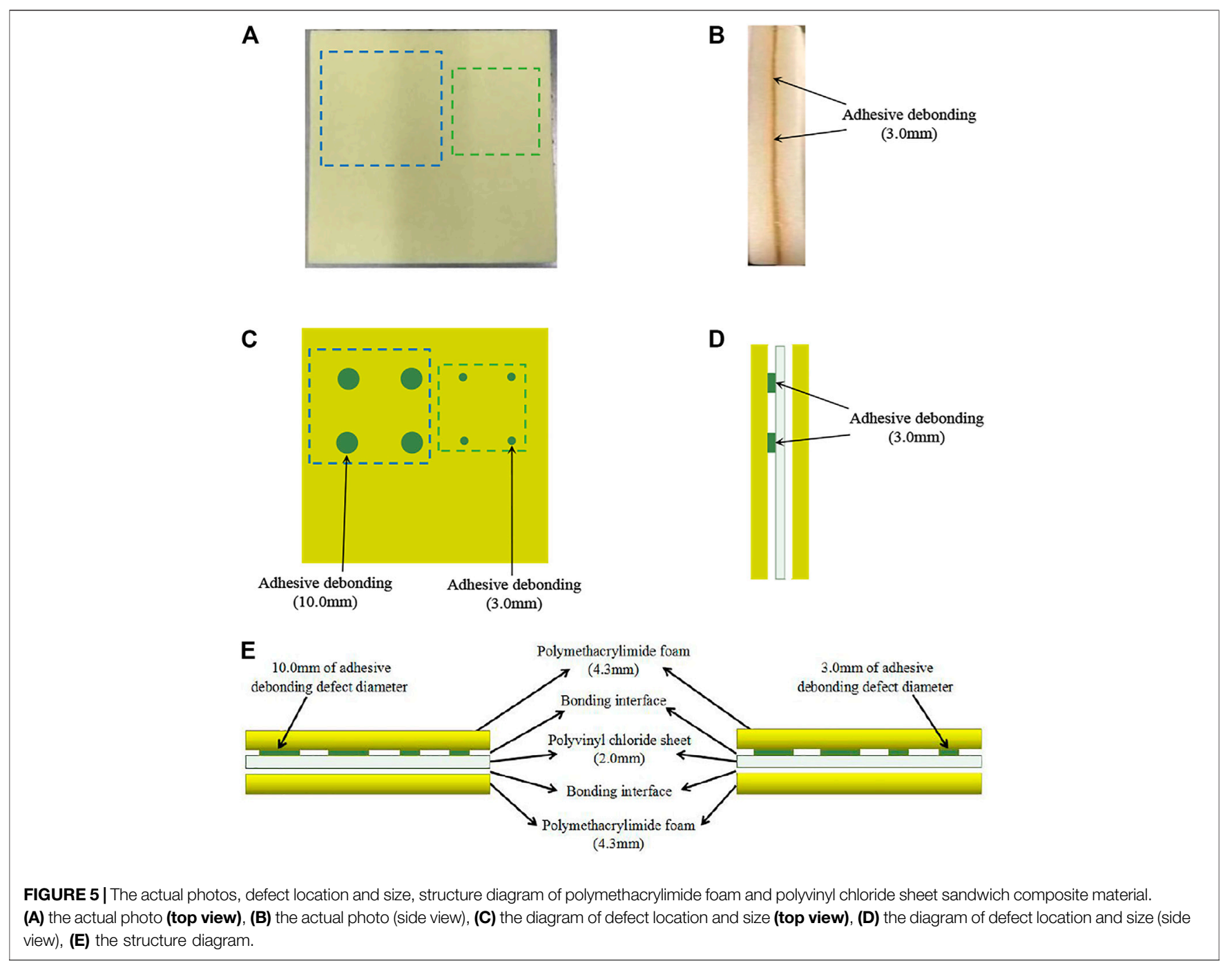

\section{Sample1: Imaging Results Analysis}

The imaging results of each method for the adhesive debonding defect in sample 1 with a diameter of $10.0 \mathrm{~mm}$ are shown in Figure 6, and the imaging results of each method for the adhesive debonding defect with a diameter of $6.0 \mathrm{~mm}$ are shown in Figure 7. Firstly, according to the observation of the original detection images of two different sizes of the adhesive debonding defects, no matter the diameter of $6.0 \mathrm{~mm}$ or $10.0 \mathrm{~mm}$, the adhesive debonding defects could not be effectively identified, only part of the defects could be vaguely observed, and the defect edges are blurred, the imaging contrast is not obvious, and the resolution of small targets is poor. Theoretically, the color distribution of the background region of the detection image should be relatively uniform, while there are differences in the color distribution of the background region in the actual detection image, with obvious noise distribution, which affects the quality of the detection image. The main reason for this phenomenon is that the energy loss of terahertz wave propagating in the measured sample is serious, and the effective reflection signal received by the detector is weak, which leads to the effective signal being submerged by noise, thus reducing the signal-noise ratio of the detected signal and reducing the imaging effect. Then observe the results of Gaussian filtering processing, although the use of this method can suppress the background noise of the detection image to some extent and enhance the contrast of the image, but the denoising is not complete, the background region still exists obvious noise distribution, unable to accurately identify the prefabricated adhesive debonding defects. Furthermore, the problem of signal distortion appears after filtering with this method, so that the effective defect information has been damaged. Finally, the results of the method proposed in this paper show that the recognition effect of the prefabricated defect region is significantly better than that of other methods. The prefabricated adhesive debonding defects are all effectively identified, and the details such as the edge of the defect are enhanced, and the contrast with the background region is improved. In addition, the noise in the background region of 

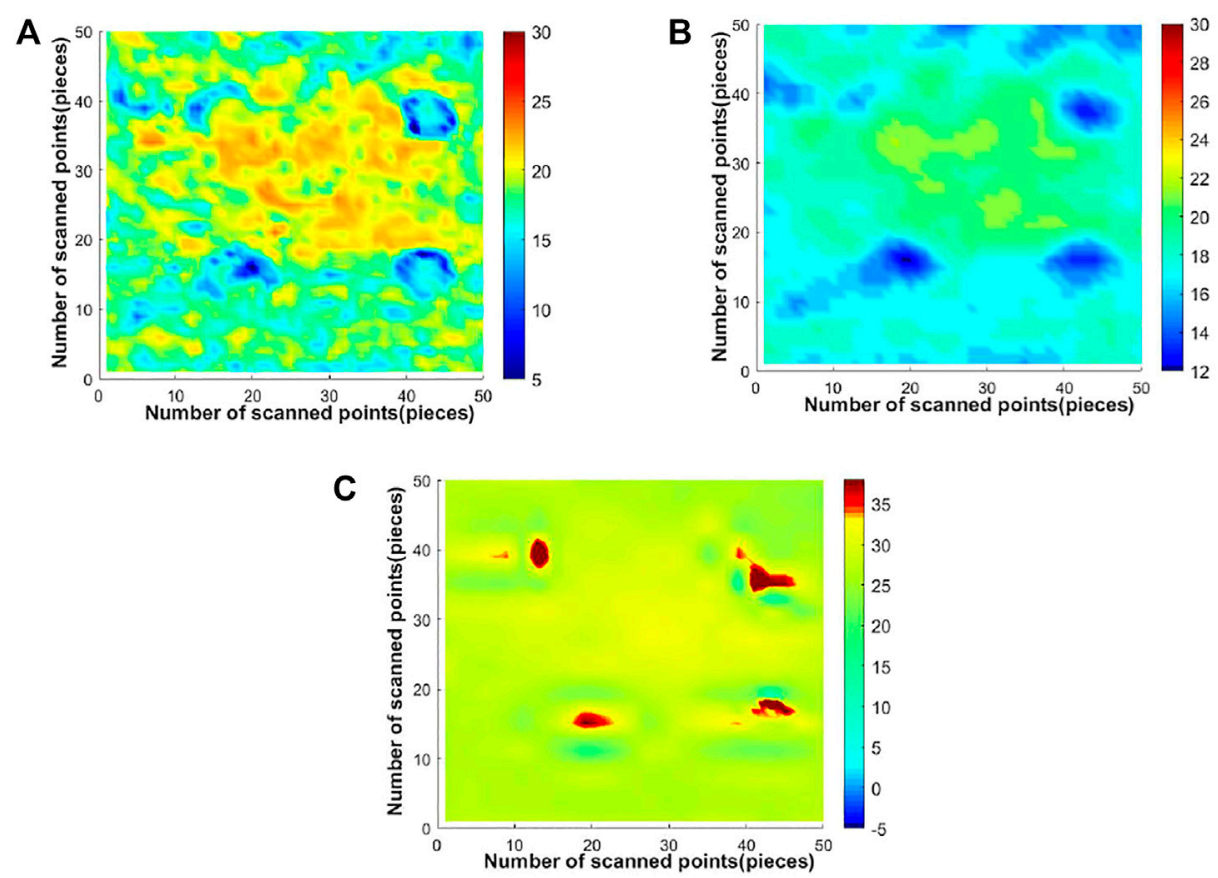

FIGURE 6 | Image of processing results of each method for adhesive debonding with a diameter of $10.0 \mathrm{~mm}$ in sample 1. (A) image of original detection, (B) Gaussian filtering, (C) method in this paper.
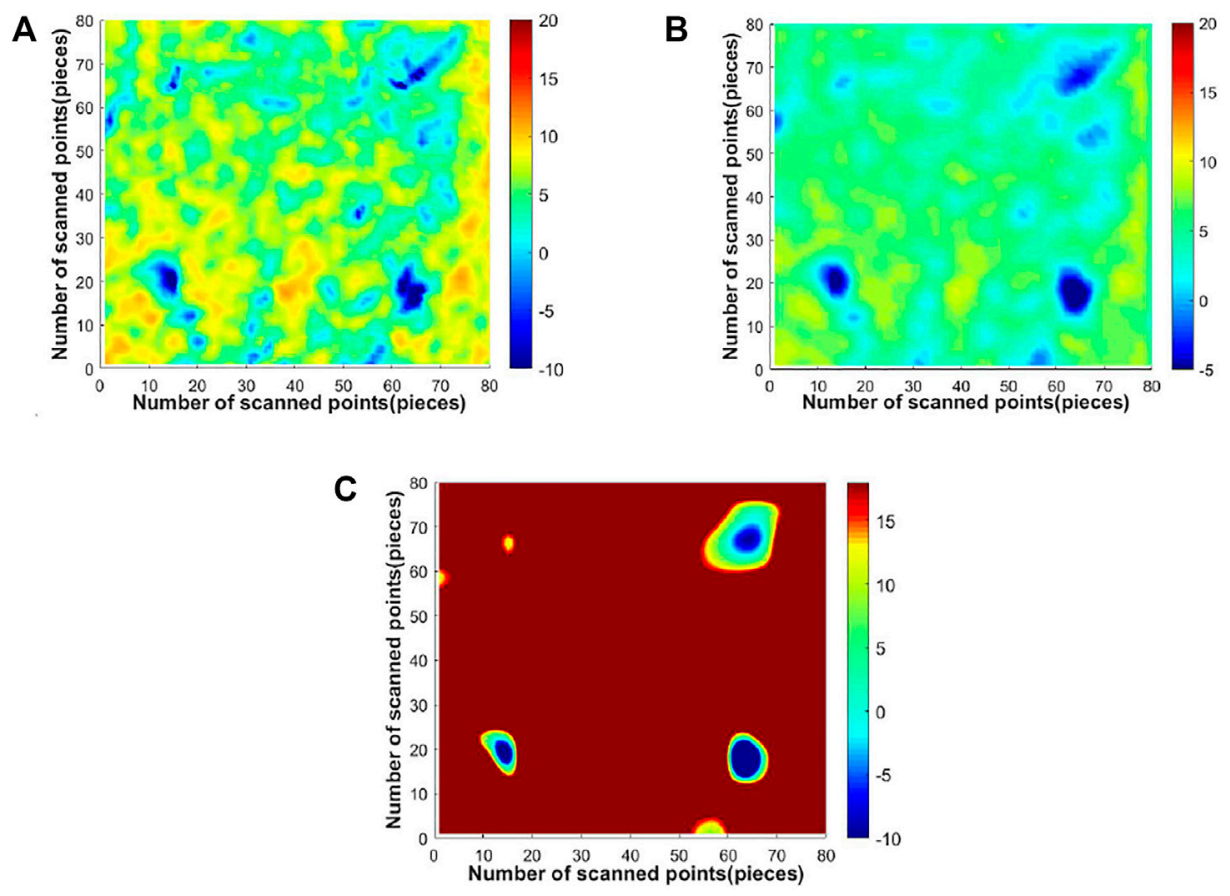

FIGURE 7 | Image of processing results of each method for adhesive debonding with a diameter of $6.0 \mathrm{~mm}$ in sample 1. (A) image of original detection, (B) Gaussian filtering, (C) method in this paper. 

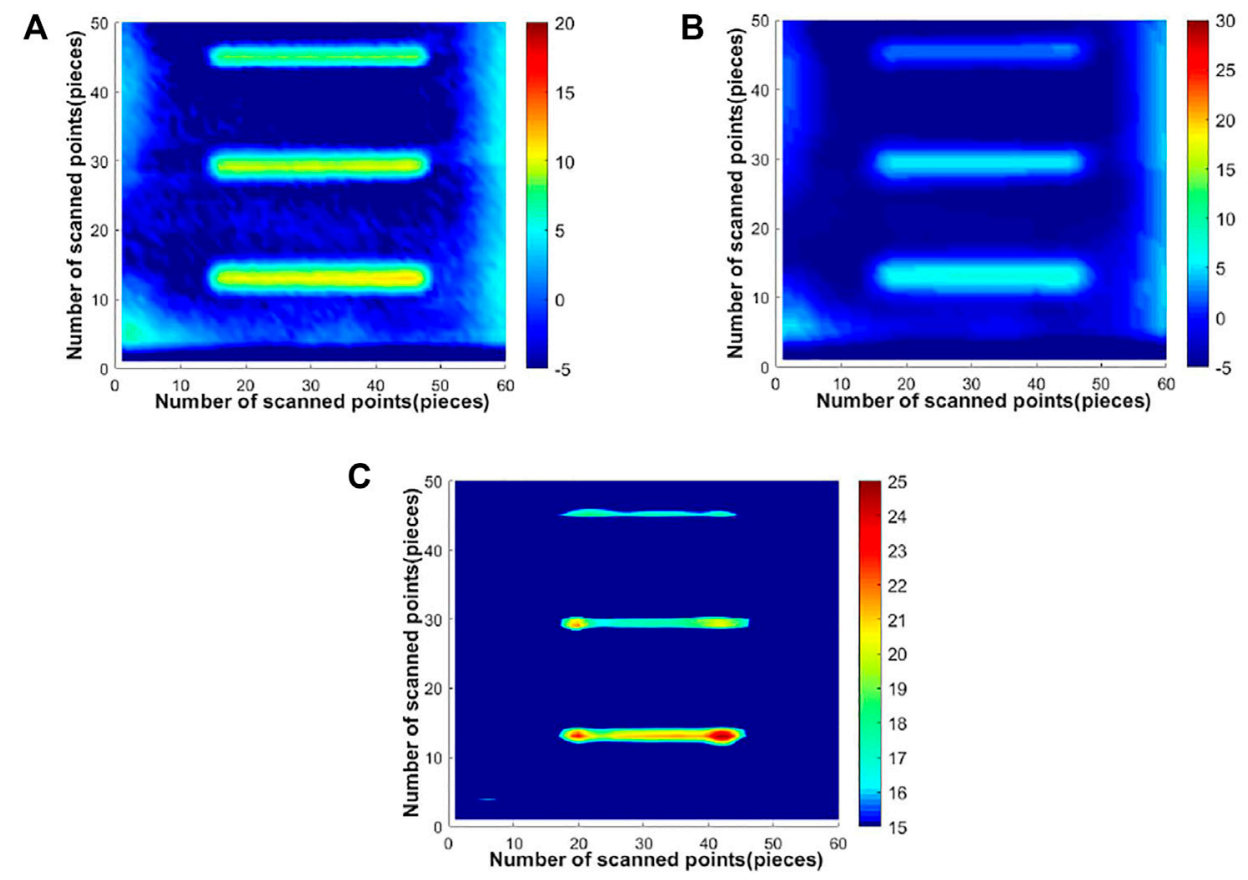

FIGURE 8 | Image of processing results of each method for delamination defects in sample 2. (A) image of original detection, (B) Gaussian filtering, (C) method in this paper.
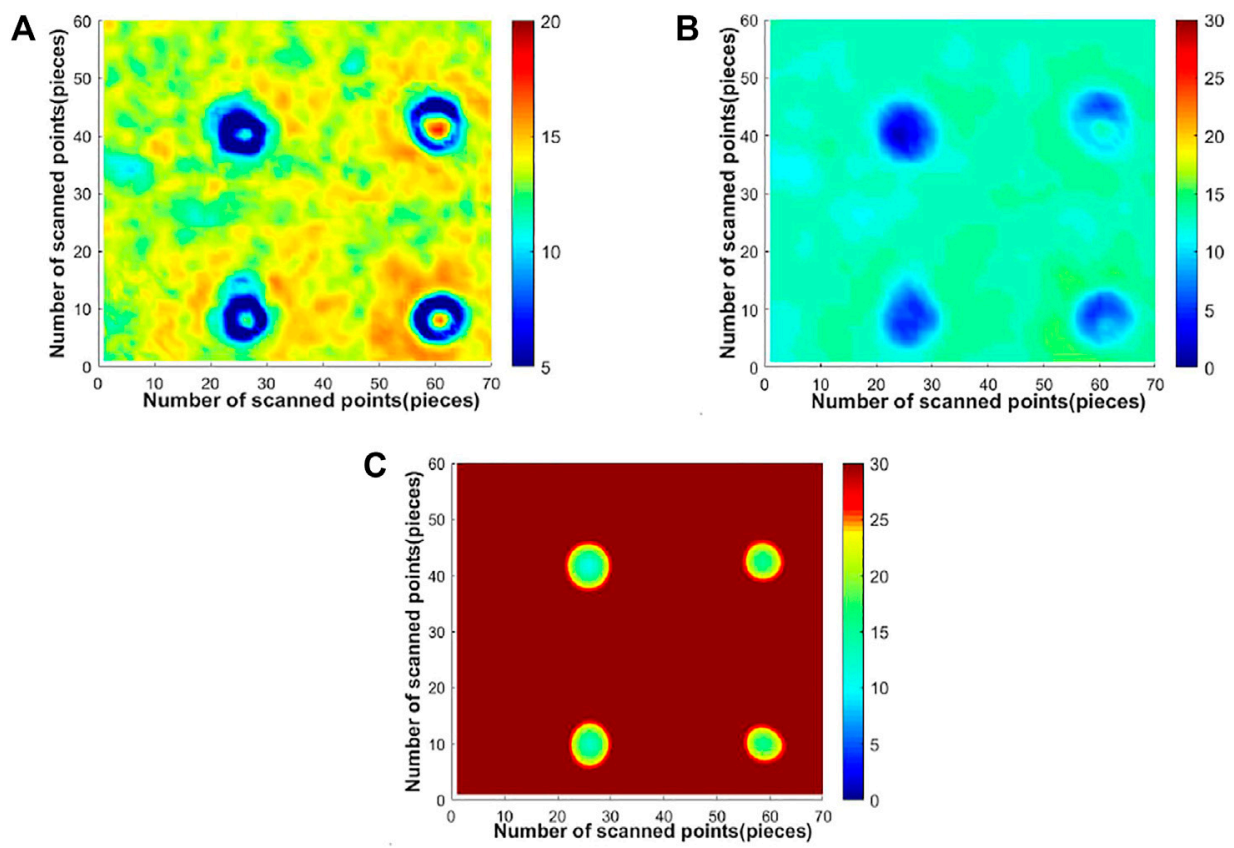

FIGURE 9 | Image of processing results of each method for adhesive debonding with a diameter of $10.0 \mathrm{~mm}$ in sample 3 . (A) image of original detection, (B) Gaussian filtering, (C) method in this paper. 

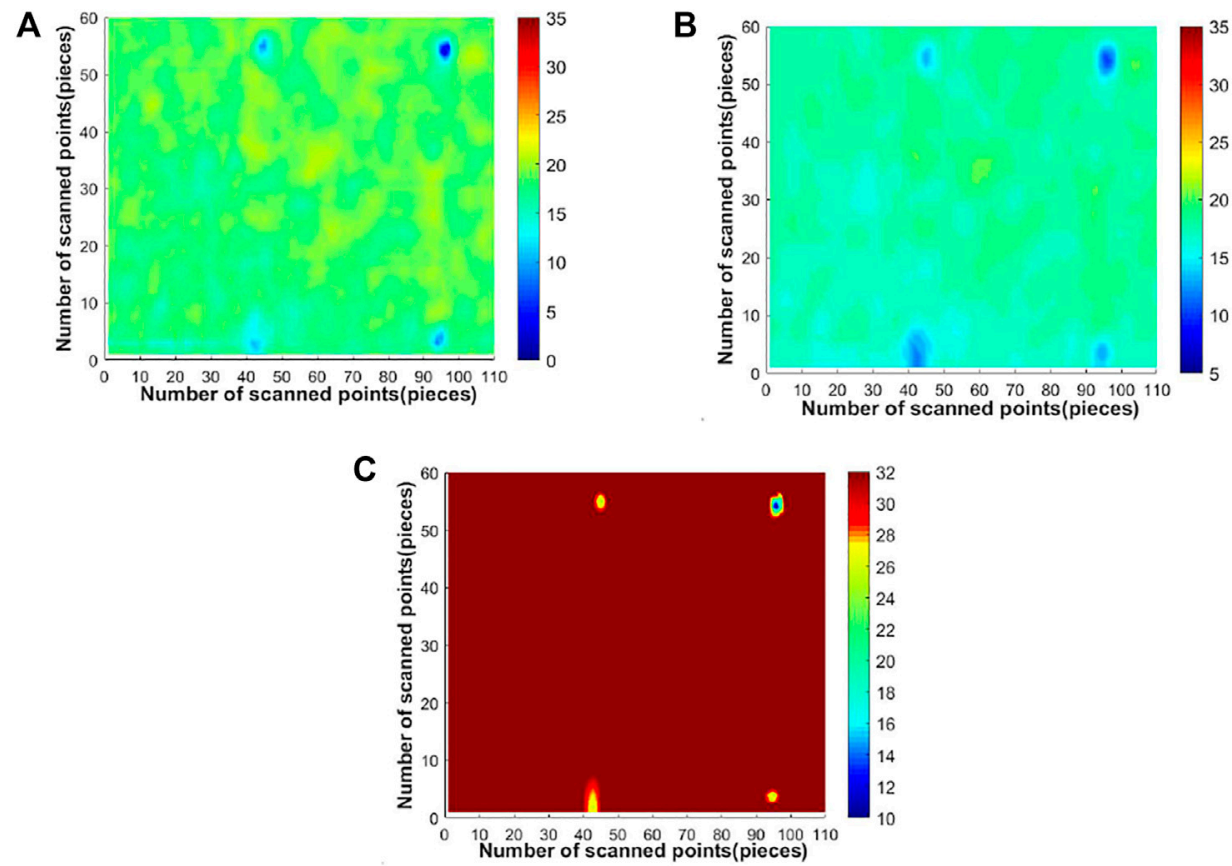

FIGURE 10 | Image of processing results of each method for adhesive debonding with a diameter of $3.0 \mathrm{~mm}$ in sample 3 . (A) image of original detection, (B) Gaussian filtering, (C) method in this paper.

TABLE 1 | Contrast results of image objective indexes between original image and images with different methods (Bold numbers indicate the optimal value for each set of data).

\begin{tabular}{|c|c|c|c|c|}
\hline Detection image & Index & Original image & Gaussian filtering & Method in this paper \\
\hline & $\mathrm{SD}$ & 3.0047 & 2.7121 & 3.6210 \\
\hline \multirow[t]{5}{*}{ Adhesive debonding defect with diameter 10.0 mm (Sample 1) } & MG & 0.0459 & 0.0198 & 0.0557 \\
\hline & IE & 6.6520 & 3.1218 & 6.7522 \\
\hline & EG & $2.8653 e+04$ & $5.3570 \mathrm{e}+03$ & $4.2509 e+04$ \\
\hline & LC & 35.6725 & 35.0706 & 54.6020 \\
\hline & $\mathrm{SD}$ & 2.9775 & 2.2973 & 13.9005 \\
\hline \multirow[t]{5}{*}{ Adhesive debonding defect with diameter 6.0 mm (Sample 1) } & MG & 0.0264 & 0.0135 & 0.0481 \\
\hline & IE & 6.7008 & 3.1171 & 8.9169 \\
\hline & $E G$ & $2.8306 e+04$ & $7.4000 e+03$ & $9.4433 e+04$ \\
\hline & LC & 9.6327 & 10.1058 & 76.1488 \\
\hline & $\mathrm{SD}$ & 6.6101 & 4.9137 & 13.3256 \\
\hline \multirow[t]{5}{*}{ Delamination defect with depth of 2.0 mm (Sample 2) } & $M G$ & 0.0618 & 0.0306 & 0.1055 \\
\hline & $\mathrm{IE}$ & 7.7592 & 4.1788 & 8.6975 \\
\hline & $E G$ & $6.5960 \mathrm{e}+04$ & $1.6507 e+04$ & $1.9495 e+05$ \\
\hline & LC & 2.4933 & 1.9407 & 2.7272 \\
\hline & $\mathrm{SD}$ & 2.4363 & 2.0684 & 8.7838 \\
\hline \multirow[t]{5}{*}{ Adhesive debonding defect with diameter 10.0 mm (Sample 3) } & $M G$ & 0.0275 & 0.0130 & 0.0308 \\
\hline & $\mathrm{IE}$ & 5.6032 & 2.2544 & 7.3732 \\
\hline & $E G$ & $2.5453 e+04$ & $5.6940 \mathrm{e}+03$ & $3.1882 e+04$ \\
\hline & LC & 24.6274 & 23.8015 & 98.5350 \\
\hline & $\mathrm{SD}$ & 1.3442 & 1.6127 & 2.6318 \\
\hline \multirow[t]{4}{*}{ Adhesive debonding defect with diameter 3.0 mm (Sample 3) } & MG & 0.0067 & 0.0080 & 0.0094 \\
\hline & IE & 5.3768 & 2.0963 & 6.2558 \\
\hline & $E G$ & $6.3414 \mathrm{e}+03$ & $9.2660 e+03$ & $1.2713 e+04$ \\
\hline & LC & 35.3572 & 34.4890 & 79.1244 \\
\hline
\end{tabular}

the image is significantly suppressed. Different from the Gaussian filtering method, the effective defect information is retained while filtering out the background noise of the image.

\section{Sample2: Imaging Results Analysis}

The imaging results of each method of the delamination defects with depth of $2.0 \mathrm{~mm}$ in sample two are shown in Figure 8. Firstly, 
the original detection image of delamination defects is observed. It can be seen that the prefabricated delamination defects can be identified by the original detection image, but the edge of the defect is blurred, the detail resolution needs to be improved, and the background region also has obvious noise distribution, which affects the quality of the detection image. After observing the results of Gaussian filtering processing, it can be seen that this method has a certain denoising effect, but the denoising effect is not obvious, there is still some residual noise, and the defect edge is still blurred, and the overall sharpness of the detection image is poor. Finally, by observing the results processed by the method in this paper, it can be seen that the overall sharpness of the detection image greatly improved, and the details such as the edge of the prefabricated delamination defects have been significantly enhanced. Moreover, the background noise of the detection image has been effectively suppressed, and the contrast between the defect and the background region has been improved, which is helpful for the accurate identification of internal defects.

\section{Sample3: Imaging Results Analysis}

The imaging results of each method for the adhesive debonding defect in sample 3 with a diameter of $10.0 \mathrm{~mm}$ are shown in Figure 9, and the imaging results of each method for the adhesive debonding defect with a diameter of $3.0 \mathrm{~mm}$ are shown in Figure 10. Firstly, the original detection images of different sizes of adhesive debonding defect in sample 3 are observed. It is found that the prefabricated adhesive debonding defect with diameters of 10.0 and $3.0 \mathrm{~mm}$ could be vaguely identified in the original detection image, but the edge of the defect is blurred and the contrast with the background area is low. In addition, the background region of the image also has obvious noise distribution, which needs further denoising and enhancement to improve the contrast of the detection image and enhance the edge of the defect and other details. By observing the detection image after Gaussian filtering, it can be seen that the prefabricated adhesive debonding defect recognition effect has been improved to some extent, the noise in the background region has been well filtered, and the contrast between the defect and the background region has also been improved to some extent. However, the detection image becomes blurred after Gaussian filtering, and the detail resolution of the small target also needs to be further improved. Finally, through the observation of the results processed by the method in this paper, it can be seen that the detection images of two kinds of adhesive debonding defect have been greatly improved in the overall sharpness, contrast and defect edge details. The prefabricated two kinds of adhesive debonding defect can be well recognized, and the background noise of the detection image has been effectively suppressed. While filtering out the background noise, the defect information is effectively retained. Compared with the original detection image and the image after Gaussian filtering, the image quality is better.

\section{OBJECTIVE EVALUATION OF RESULTING IMAGES}

In order to accurately evaluate the image processed by each method, this paper also uses five objective evaluation indexes, namely standard deviation (SD), mean gradient (MG), information entropy (IE), energy gradient (EG) and local contrast (LC), to objectively evaluate the image processing results [25-27].

\section{Standard Deviation}

Standard deviation refers to the degree of dispersion of image pixel gray value relative to the mean value. If the standard deviation is larger, it indicates that the gray scale in the image is more dispersed and the image quality is better. Its calculation formula is

$$
S D=\sqrt{\frac{1}{G R} \sum_{i=1}^{G} \sum_{j=1}^{R}(F(i, j)-u)^{2}}
$$

where $G$ and $R$ represent the sizes of the image, $F(i, j)$ represents the gray value of pixel $F(i, j)$ corresponding to image $F$, and $u$ represents the average gray value of all pixels in the image.

\section{Mean Gradient}

The mean gradient represents the ability of image to express details, which is a measure of image sharpness. Usually, the greater the mean gradient is, the more the details of the image are, and the higher the sharpness is. The calculation formula of the mean gradient is as follows

$$
M G=\frac{1}{G R} \sum_{i=1}^{G} \sum_{j=1}^{R} \sqrt{\left[\frac{\partial f(x, y)}{\partial x}\right]^{2}+\left[\frac{\partial f(x, y)}{\partial y}\right]^{2}}
$$

where $\frac{\partial f(x, y)}{\partial x}$ represents the gradient in the horizontal direction, and $\frac{\partial f(x, y)}{\partial y}$ represents the gradient in the vertical direction.

\section{Information Entropy}

Information entropy is an effective method to evaluate information content in images. For an image with gray scale in the range of $[0, \mathrm{~L}-1]$, the expression of information entropy is

$$
I E=-\sum_{i=0}^{L-1} p\left(s_{i}\right) \log _{2} p\left(s_{i}\right)
$$

Where $p\left(s_{i}\right)$ is the probability of gray level $s_{i}$, the greater the information entropy indicates that the image contains more details.

\section{Energy Gradient}

Image sharpness is an important index to measure the quality of the image, which can better correspond to the subjective feelings of people. The energy gradient can evaluate the image sharpness in real time, and the greater the energy gradient value of the image, the better the image sharpness. The definition of the function is as follows

$$
\begin{aligned}
E G= & \sum_{x=1}^{G} \sum_{y=1}^{R}\left(|f(x+1, y)-f(x, y)|^{2}\right. \\
& \left.+|f(x, y+1)-f(x, y)|^{2}\right)
\end{aligned}
$$


Where $f(x, y)$ represents the gray value of image $f$ at the corresponding pixel $(x, y)$.

\section{Local Contrast}

The local contrast of the image can reflect the dynamic range of the gray distribution of the image pixels. The larger the dynamic range is, the higher the contrast is. The formula for calculating the local contrast is

$$
L C=\frac{1}{G R} \sum_{x=1}^{G} \sum_{y=1}^{R} \frac{I_{x, y}^{\max }-I_{x, y}^{\min }}{I_{x, y}^{\max }+I_{x, y}^{\min }+C}
$$

Where $I_{x, y}^{\max }$ and $I_{x, y}^{\min }$ represent the maximum and minimum pixel gray values in a sub-block centered on pixel $(x, y)$, respectively. In this paper, the size of the sub-block is $5 \times 5$. In order to avoid the case of denominator $0, C$ is a smaller constant with a value of 0.1 .

The calculation results of objective evaluation indexes of different methods are shown in Table 1. It can be seen from the table that the standard deviation, mean gradient, information entropy, energy gradient and local contrast of the five objective evaluation index data are higher than the corresponding values of the original detection image and the detection image after Gaussian filtering. It shows that the method in this paper has obvious advantages in contrast enhancement and level of detail and sharpness improvement.

\section{CONCLUSION}

Aiming at the degradation phenomena of terahertz detection images, such as low contrast, poor resolution of small targets and blurred details, this paper proposes a novel method for the enhancement of composite materials' terahertz image using unsharp masking and guided filtering technology. Through the imaging detection of a variety of composite materials, denoise and enhance the imaging by using this novel method, the experimental results and evaluation results

\section{REFERENCES}

1. Siegel PH. Terahertz Technology. IEEE Trans Microwave Theor Techn. (2002) 50(3):910-28. doi:10.1109/22.989974

2. Kawase K, Shibuya T, Hayashi Si., and Suizu K. THz Imaging Techniques for Nondestructive Inspections. Comptes Rendus Physique (2010) 11(7-8):510-8. doi:10.1016/j.crhy.2010.04.003

3. Ferguson B, and Abbott D. De-noising Techniques for Terahertz Responses of Biological Samples. Microelectron J (2001) 32(12):943-53. doi:10.1016/S00262692(01)00093-3

4. Kowalski M, Kastek M, Walczakowski M, Palka N, and Szustakowski M. Passive Imaging of Concealed Objects in Terahertz and Long-Wavelength Infrared. Appl Opt (2015) 54(13):3826-33. doi:10.1364/AO.54.003826

5. Hua Zhong H, Yongwei Li YW, and Jiao LC. SAR Image Despeckling Using Bayesian Nonlocal Means Filter with Sigma Preselection. IEEE Geosci Remote Sens Lett (2011) 8(4):809-13. doi:10.1109/LGRS.2011.2112331

6. Buades A, Coll B, and Morel JM. A Review of Image Denoising Algorithms, with a New One. Multiscale Model Simul (2005) 4(2):490-530. doi:10.1137/040616024

7. Buades A, Coll B, and Morel J-M. A Non-local Algorithm for Image Denoising. In: IEEE Computer Society Conference on Computer Vision and Pattern show that the proposed method can effectively filter out the noise in the detection signal, improve the image contrast and resolution, and enhance the details of the detection image, which is conducive to the wider application of terahertz technology in the field of nondestructive testing of composite materials. In the future research, the existing methods are further improved to find a new method to achieve the size of the identified defect close to the actual size on the basis of improving the contrast and detail resolution of the terahertz detection image.

\section{DATA AVAILABILITY STATEMENT}

The raw data supporting the conclusion of this article will be made available by the authors, without undue reservation.

\section{AUTHOR CONTRIBUTIONS}

ZL: Methodology, Experiment, Validation, Investigation, Writing-Original Draft; JZ: Supervision, Investigation; YMZ: Supervision, Methodology, Writing-Reviewing and Editing; ZH: Experiment; ZX: Experiment; YZZ: Experiment; CY: Sample preparation; WH: Experiment, Supervision; CZ: Supervision, Funding acquisition.

\section{FUNDING}

This research was supported by the Beijing Advanced Innovation Center for Imaging Theory and Technology Scientific Research Funds (008/19530012003), the Capital Normal University Development Funds by Category-Physics Department-Practice base Projects for Degree Study Program (008-2155089).

Recognition (CVPR'05); 2005 Jun 20-25; San Diego, CA, USA. IEEE (2005) doi:10.1109/CVPR.2005.38

8. Shi YX, and Li JS. Based on Double Threshold Canny Equalization Algorithm for Terahertz Image Enhancement. Spectrosc Spect Anal (2018) 38(6):1680-3. doi:10.3964/j.issn.1000-0593(2018)06-1680-04

9. He K, Sun J, and Tang X. Guided Image Filtering. IEEE Trans Pattern Anal Mach Intell (2013) 35(6):1397-409. doi:10.1109/TPAMI.2012.213

10. Liu N, and Zhao D. Detail Enhancement for High-Dynamic-Range Infrared Images Based on Guided Image Filter. Infrared Phys Technol (2014) 67:138-47. doi:10.1016/j.infrared.2014.07.013

11. Zhou B, Luo Y, Yang M, Chen B, Wang M, Peng L, et al. An Improved Adaptive Detail Enhancement Algorithm for Infrared Images Based on Guided Image Filter. J Mod Opt (2019) 66(1):33-46. doi:10.1080/ 09500340.2018.1511861

12. Li Y, Hu W, Zhang X, Zhao Y, Ni J, and Ligthart LP. A Non-linear Correction Method for Terahertz LFMCW Radar. IEEE Access (2020) 8:102784-94. doi:10.1109/ACCESS.2020.2998602

13. Dong J, Kim B, Locquet A, McKeon P, Declercq N, and Citrin DS. Nondestructive Evaluation of Forced Delamination in Glass FiberReinforced Composites by Terahertz and Ultrasonic Waves. Composites B Eng (2015) 79:667-75. doi:10.1016/j.compositesb.2015.05.028 
14. Maraun D, and Kurths J. Cross Wavelet Analysis: Significance Testing and Pitfalls. Nonlin Process. Geophys (2004) 11(4):505-14. doi:10.5194/npg-11505-2004

15. Mallat S, and Hwang WL. Singularity Detection and Processing with Wavelets. IEEE Trans Inform Theor (1992) 38(2):617-43. doi:10.1109/18.119727

16. Sappa A, Carvajal J, Aguilera C, Oliveira M, Romero D, and Vintimilla B. Wavelet-Based Visible and Infrared Image Fusion: A Comparative Study. Sensors (2016) 16(6):861. doi:10.3390/s16060861

17. Mallat SG. A Theory for Multiresolution Signal Decomposition: the Wavelet Representation. IEEE Trans Pattern Anal Machine Intell (1989) 11(7):674-93. doi: $10.1109 / 34.192463$

18. González-Audícana M, Otazu X, Fors O, and Seco A. Comparison between Mallat's and the 'à trous' discrete wavelet transform based algorithms for the fusion of multispectral and panchromatic images. Int J Remote Sens (2005) 26(3):595-614. doi:10.1080/01431160512331314056

19. Ramponi G. A Cubic Unsharp Masking Technique for Contrast Enhancement. Signal Process. (1998) 67(2):211-22. doi:10.1016/S01651684(98)00038-3

20. Polesel A, Ramponi G, and Mathews VJ. Image Enhancement via Adaptive Unsharp Masking. IEEE Trans Image Process (2000) 9(3): 505-10. doi:10.1109/83.826787

21. Xing L, Cui H-L, Shi C, Zhang Z, Zhang J, Chang T, et al. Nondestructive Examination of Polymethacrylimide Composite Structures with Terahertz Time-Domain Spectroscopy. Polym Test (2017) 57:141-8. doi:10.1016/ j.polymertesting.2016.11.022

22. Frydrych I, Xu F, Duan Mu QD, Li LJ, Yang D, and Xia B. Nondestructive Evaluation of Rubber Composites Using Terahertz Time Domain Spectroscopy. Fibres Text East Europe (2018) 26(1):67-72. doi:10.5604/01.3001.0010.7799

23. Ospald F, Zouaghi W, Beigang R, Matheis C, Jonuscheit J, Recur B, et al. Aeronautics Composite Material Inspection with a Terahertz TimeDomain Spectroscopy System. Opt Eng (2013) 53(3):031208. doi:10.1117/1.OE.53.3.031208
24. Walker J, and Richter J. Nondestructive Evaluation of Foam Insulation for the External Tank Return to Flight. In: 42nd AIAA/ASME/SAE/ASEE Joint Propulsion Conference \& Exhibit, Sacramento, California (2006). doi:10.2514/6.2006-4601

25. Liu J, Zhou C, Chen P, and Kang C. An Efficient Contrast Enhancement Method for Remote Sensing Images. IEEE Geosci Remote Sens Lett (2017) 14(10):1715-9. doi:10.1109/LGRS.2017.2730247

26. Wang Y, and Pan Z. Image Contrast Enhancement Using Adjacent-BlocksBased Modification for Local Histogram Equalization. Infrared Phys Technol (2017) 86:59-65. doi:10.1016/j.infrared.2017.08.005

27. Subbarao M, Choi T, and Nikzad A. Focusing Techniques. Opt Eng (1993) 32(11):2824-36. doi:10.1117/12.147706

Conflict of Interest: Author CY is employed by Shenzhen Kuang-Chi Advanced Technology Co., Ltd.

The remaining authors declare that the research was conducted in the absence of any commercial or financial relationships that could be construed as a potential conflict of interest.

Publisher's Note: All claims expressed in this article are solely those of the authors and do not necessarily represent those of their affiliated organizations, or those of the publisher, the editors and the reviewers. Any product that may be evaluated in this article, or claim that may be made by its manufacturer, is not guaranteed or endorsed by the publisher.

Copyright (c) $2021 \mathrm{Li}, \mathrm{Zuo}, \mathrm{Zhao}, \mathrm{Han}, \mathrm{Xu}$, Zhao, Yang, Hu and Zhang. This is an open-access article distributed under the terms of the Creative Commons Attribution License (CC BY). The use, distribution or reproduction in other forums is permitted, provided the original author(s) and the copyright owner(s) are credited and that the original publication in this journal is cited, in accordance with accepted academic practice. No use, distribution or reproduction is permitted which does not comply with these terms. 